\title{
Psychedelics and the science of self-experience
}

\author{
Matthew M. Nour and Robin L. Carhart-Harris
}

\section{Summary}

Altered self-experiences arise in certain psychiatric conditions, and may be induced by psychoactive drugs and spiritual/religious practices. Recently, a neuroscience of selfexperience has begun to crystallise, drawing upon findings from functional neuroimaging and altered states of consciousness occasioned by psychedelic drugs. This advance may be of great importance for psychiatry.

\section{Declaration of interest \\ None.}

\section{Copyright and usage}

(c) The Royal College of Psychiatrists 2017.
Matthew Nour (pictured) is an NIHR Academic Clinical Fellow in Psychiatry at King's College London and South London and Maudsley NHS Foundation Trust. Robin Carhart-Harris is Head of Psychedelic Research at the Centre for Neuropsychopharmacology, Imperial College London, where he studies the neuroscience and therapeutic potential of psychedelic drugs.

\section{Defining the self}

The experience of a coherent and well-circumscribed 'self' is a cardinal feature of human waking consciousness. ${ }^{1}$ Despite our ready use of personal pronouns such as 'me' and 'I', the nature of self remains one of the central questions confronting philosophy, psychology and cognitive science, and continues to defy simple definition. Philosophers distinguish between two notions of self. At one level, self-experience comprises the experience of a unified personal identity, associated with our own individual life narrative, enduring character traits, life goals and a hierarchy of personal values. This 'narrative self' may be understood as a cognitive theoretical construct - an object of knowledge, emerging from the stories that we tell, and are told, about ourselves. At a more fundamental level, first-person experience is saturated by immediate aspects of self, which include the unity and familiarity of experience, a sense of embodiment and a sense of agency. This 'minimal self' forms the subject of experience, and is independent of the contingent facts that give substance to the narrative self. ${ }^{2}$

\section{Self in neuroscience}

The heterogeneous and multilayered nature of self-experience presents a challenge for an emerging neuroscience of selfconsciousness, which ultimately seeks to identify underlying neuronal correlates and mechanisms. The neuronal correlates of self have been investigated using tasks that seek to identify brain regions that display increased activity (typically measured using functional magnetic resonance imaging (fMRI)) during processing of self-specific stimuli (for example images, voices, trait adjectives), compared with stimuli related to others. A recent quantitative meta-analysis, pooling data from nearly 1500 participants, implicated activity in cortical midline brain regions as a neuronal correlate of self-experience. Specifically, activity in the perigenual anterior cingulate cortex was associated with processing of self-specific stimuli, compared with non-self stimuli. $^{3}$

Interestingly, the brain regions implicated in self-processing show anatomical overlap with important nodes of the default mode network (DMN). ${ }^{3}$ The DMN is a network of mostly cortical brain regions that appears to be involved in high-level psychological functions including introspection and autobiographical memory. ${ }^{1,3}$ The brain regions comprising the DMN act as 'connector hubs' within the brain, displaying high metabolic demands and inter-region functional connectivity at rest.

The studies discussed above may shed light on the neuronal mechanisms underlying self-attribution to physical and mental features (approaching the self as an object of attribution), but are not well suited to identify neuronal correlates of minimal self-experience (self as knowing subject). ${ }^{2}$ Phenomenological features of minimal self-experience, such as self-ownership and self-agency, are present in the structure of first-person subjective experience itself, ${ }^{4}$ suggesting that their neuronal correlates may be found in the very building blocks of neuronal computation.

Theoretical considerations suggest that the brain is perpetually engaged in generating predictions about the 'state of the world', and testing these predictions against incoming sensory signals. ${ }^{1,4}$ Perception and action depend on neuronal mechanisms that minimise the mismatch between predicted and actual incoming signals. This process of mismatch-minimisation would theoretically imbue perception and action with a pre-reflective feeling of familiarity, self-ownership and self-agency, making it a good candidate for the neuronal correlate of minimal self-experience. ${ }^{2,5}$ It is conceivable that the development of more 'cognitive' facets of self, such as personal narrative, may be predicated on minimal self-experiences.

\section{Self-disturbance}

Distortions of self-experience are a central feature of a number of altered states of consciousness, such as the psychedelic state and the mystical experience. ${ }^{1,6-9}$ In these contexts the experience of a reduction in the normally well-circumscribed experience of self has been termed 'ego-dissolution', and is related to a feeling of increased unity with others and one's surroundings. ${ }^{1,6-8}$

Abnormalities of self-consciousness have also been described in a number of mental health conditions. ${ }^{10}$ A disrupted sense of self was considered to be a core phenomenological feature of early and acute psychosis. ${ }^{4}$ Kraepelin, for example, wrote of a 'disunity 
of consciousness' in schizophrenia and Bleuler wrote of a slackening of the associative links that tie together thoughts, perceptions and affects. ${ }^{4}$ More recently, it has been proposed that a central phenomenological alteration in schizophrenia is an instability of 'pre-reflective self-awareness', which has close parallels to conceptions of minimal self-experience, and is related to a feeling of immersion in a social world. ${ }^{4,11}$ Recent efforts to operationalise self-disturbance in schizophrenia, using the Examination of Anomalous Self-Experience (EASE) questionnaire, suggest that this feature may predict clinical trajectory of firstepisode psychosis. ${ }^{11}$

By contrast, the depressed state is characterised by an increase in self-focus, and an inability to disengage from self-referential ruminative thought. Intriguingly, individuals with depression show abnormal activity in the perigenual anterior cingulate cortex, a region that has been shown to exhibit increased activity for self-specific stimuli. ${ }^{3,10}$ More generally, it is noteworthy that both schizophrenia and depression are characterised by abnormal DMN resting-state activity. ${ }^{10}$

\section{Psychedelics as a window into the self}

Given the prominence of self-experience in normal waking consciousness, and the prevalence of self-disturbance in certain psychiatric conditions, a greater understanding of the neuronal correlates of self-experience is highly desirable. Psychedelic drugs (serotonin-2A $\left(5-\mathrm{HT}_{2 \mathrm{~A}}\right)$ receptor agonists) reliably perturb selfconsciousness and occasion ego-dissolution experiences in a reversible, transient and dose-dependent manner. ${ }^{8}$ These substances therefore provide a fruitful avenue for research into the neuronal correlates of normal and abnormal self-consciousness. ${ }^{1,7,12}$ In recent years, several studies have examined the neuronal correlates of ego-dissolution experiences occasioned by the classic psychedelic drugs psilocybin (the active agent in 'magic mushrooms') and lysergic acid diethylamide (LSD) using fMRI and magnetoencephalography (MEG). These studies reveal that psychedelics decrease the integrity of important resting-state brain networks associated with the sense of self (such as the DMN) as well as the degree of segregation between these and other brain networks. As a result, psychedelics occasion a more globally unified mode of brain function. ${ }^{1}$ Importantly, these changes (i.e. DMN disintegration and whole-brain integration) correlate with participants' ratings of ego-dissolution. ${ }^{6,7}$ A similar pattern of decreased segregation between functional networks has also been observed in meditators during maintenance of non-dual awareness (where equal attention is given to events 'inside and outside' the body) compared with maintenance of focused attention on a single object. ${ }^{13}$

A recent MEG study demonstrated that ego-dissolution experiences occasioned by psilocybin correlate highly with decreased alpha power (a marker of locally synchronous activity) in the posterior cingulate cortex, arguably the core DMN 'hub' region. ${ }^{12}$ Moreover, two recent studies found that the degree of ego-dissolution occasioned by LSD was correlated with increased whole-brain integration, ${ }^{7}$ and inversely correlated with DMN network integrity, functional connectivity between the parahippocampus and retrosplenial cortex, and oscillatory power in the delta and alpha frequency bands (for example in posterior cingulate cortex) ${ }^{6}$ nicely corroborating the earlier findings with psilocybin.

Together, these studies suggest that abnormalities in selfexperience may arise when the normal modular organisation of the brain's resting-state functional networks is disrupted. One limitation of these studies, however, is that they did not use independently validated measures of ego-dissolution. To rectify this limitation we recently validated a new eight-item self-report assessment of ego-dissolution, the Ego-Dissolution Inventory, ${ }^{8}$ with the aim of demonstrating the construct validity of egodissolution, and increasing the reliability of its measurement in future studies.

\section{Therapeutic implications}

An understanding of the neuronal correlates of abnormal selfexperience is of great relevance for our understanding and treatment of certain psychiatric conditions. In recent years the therapeutic utility of psychedelic drugs in anxiety, addiction and mood disorders has received increased attention. ${ }^{14}$ Several small studies suggest that psychedelic substances can produce improvements in these conditions when given in controlled therapeutic environments. Recently, a small open-label pilot study of psilocybin in treatment-resistant depression demonstrated improvements in self-reported depressive symptoms at 3-month follow-up. ${ }^{15}$ The precise mechanism by which psychedelics can occasion positive and lasting changes in mood, attitudes and behaviours is deserving of further investigation. It is worth noting, however, that one influential model of psychedelic therapy places great therapeutic importance on the experience of ego-dissolution, and loss of boundaries between the subject and the objective world, with ensuing feelings of unity' ${ }^{8}$

In non-clinical populations, previous work has established that mystical experiences occasioned by psilocybin (which are closely related to ego-dissolution experiences ${ }^{8}$ ) correlate positively with well-being, increases in the personality trait of openness and the meaningfulness/spiritual significance of the experience. ${ }^{8,14}$ It should be noted, however, that these clinical and non-clinical studies have strict exclusion criteria, small sample sizes and relatively short follow-up periods. Consequently, further research is required to adequately test the proposed link between egodissolution/mystical experiences and positive changes in mood, attitudes and behaviour.

\section{Conclusions}

A neuroscience of self-experience is of great importance for neuroscience in general, and for our understanding and treatment of certain psychiatric conditions in particular. Classic psychedelic drugs can occasion transient and dose-dependent reductions in the normally well-circumscribed experience of self (termed egodissolution), providing a valuable tool with which to investigate the neuronal correlates of normal and abnormal self-experience. More speculatively, the ability of psychedelics to occasion egodissolution experiences may be of clinical utility when used in controlled therapeutic environments.

Matthew M. Nour, BA, BM BCh, Institute of Psychiatry Psychology \& Neuroscience, King's College London, London; Robin L. Carhart-Harris, BSC, MA, PhD, Centre for Neuropsychopharmacology, Division of Brain Sciences, Faculty of Medicine, Imperial College London, London, UK

Correspondence: Matthew M. Nour, Department of Psychological Medicine, King's College Hospital, Denmark Hill, London SE5 9RS, UK. Email:

matthew.nour@kcl.ac.uk

First received 17 May 2016, final revision 19 Oct 2016, accepted 4 Dec 2016

\section{Funding}

M.M.N. is funded by the National Institute for Health Research. R.L.C.-H. is funded by the Mosley Foundation. 


\section{References}

1 Carhart-Harris RL, Leech R, Hellyer PJ, Shanahan M, Feilding A, Tagliazucchi E, et al. The entropic brain: a theory of conscious states informed by neuroimaging research with psychedelic drugs. Front Hum Neurosci 2014; 8: 20.

2 Christoff $K$, Cosmelli D, Legrand $D$, Thompson E. Specifying the self for cognitive neuroscience. Trends Cogn Sci 2011; 15: 104-12.

3 Qin $P$, Northoff G. How is our self related to midline regions and the default-mode network? Neuroimage 2011; 57: 1221-33.

4 Nour MM, Barrera A. Schizophrenia, subjectivity, and mindreading. Schizophr Bull 2015; 41: 1214-9.

5 Seth AK, Suzuki K, Critchley HD. An interoceptive predictive coding model of conscious presence. Front Psychol 2012; 2: 1-16.

6 Carhart-Harris RL, Muthukumaraswamy S, Roseman L, Kaelen M, Droog W, Murphy $K$, et al. Neural correlates of the LSD experience revealed by multimodal neuroimaging. Proc Natl Acad Sci USA 2016; 113: 4853-8.

7 Tagliazucchi E, Roseman L, Kaelen M, Orban C, Muthukumaraswamy SD, Murphy $\mathrm{K}$, et al. Increased global functional connectivity correlates with LSD-induced ego dissolution. Curr Biol 2016; 26: 1043-50.
8 Nour MM, Evans L, Nutt D, Carhart-Harris RL. Ego-dissolution and psychedelics: validation of the Ego-Dissolution Inventory (EDI). Front Hum Neurosci 2016; 10: 269.

9 Grof S. LSD Psychotherapy. Hunter House Publishers, 1980.

10 Northoff G. How is our self altered in psychiatric disorders? A neurophenomenal approach to psychopathological symptoms. Psychopathology 2014; 47: 365-76.

11 Nordgaard J, Parnas J. Self-disorders and the schizophrenia spectrum: a study of 100 first hospital admissions. Schizophr Bull 2014; 40: 1300-7.

12 Muthukumaraswamy SD, Carhart-Harris RL, Moran RJ, Brookes MJ, Williams TM, Errtizoe D, et al. Broadband cortical desynchronization underlies the human psychedelic state. J Neurosci 2013; 33: 15171-83.

13 Josipovic Z, Dinstein I, Weber J, Heeger DJ. Influence of meditation on anticorrelated networks in the brain. Front Hum Neurosci 2012; 5: 183.

14 Nichols DE. Psychedelics. Pharmacol Rev 2016; 68: 264-355.

15 Carhart-harris RL, Bolstridge M, Rucker J, Day CMJ, Erritzoe D, Kaelen M, et al. Psilocybin with psychological support for treatment-resistant depression: an open-label feasibility study. Lancet Psychiatry 2016; 3: 619-27.

\section{Aidan Collins}

Dum Spiro Spero ('Whilst I breathe, I hope'). This was the motto of the Dillons of Lismullen, Co. Meath in Ireland. So it was for Laura Maud Dillon, born on 1 May 1915, the only daughter of Lt Robert Arthur Dillon (RN) and Laura Maud Reese. Her father Robert Dillon was the younger brother and heir to Sir John Fox Dillon 7th Baronet of Lismullen of Lismullen Park, now the site of the Lismullin (sic) Institute, a Retreat Centre operated by Opus Dei.

Laura spent her childhood between the family estate at Lismullen near Tara, Co. Meath and Folkestone, Kent before entering St Anne's College in Oxford for her undergraduate degree. A tall, athletic, rather androgynous woman, she represented Oxford in rowing and was president of the Ladies Rowing Club there, taking a Blue in 1938. Her brother Robert William became the 8th Baronet in 1925 .

Never feeling comfortable with her sexuality, Laura experimented with the male hormone testosterone from 1939 and spent the war years adopting male societal roles before coming to the attention of the pioneering plastic surgeon Harold Gillies, who agreed to consider her case. She continued testosterone treatment, underwent bilateral mastectomies and in 1944 had her birth certificate amended, becoming Laurence Michael Dillon.

Michael Dillon became a medical student in Trinity College, Dublin in 1944. Between 1946 and 1949 he underwent 13 gender reassignment surgeries. In 1946 he published Self: A Study in Endocrinology and Ethics, which was the first work to address the difficulties associated with transgender issues. Self brought Dillon to the attention of Robert (later Roberta) Cowell, who would become the first to undergo male to female gender reassignment. Dillon himself performed the orchidectomy (testicular amputation) on Cowell, although he was still only a medical student. Their relationship became an affair, although ultimately Cowell rejected Dillon.

Dillon represented Trinity in men's rowing and after qualification in 1951 he became a ship's doctor. This suited his wish for relative obscurity. He made an error in this regard by producing his amended birth certificate to Debrett's, the journal listing aristocratic titles and lines of inheritance. In 1958 Dillon was listed as the next male in line for the baronetcy at Lismullen and the discrepancy between Debrett's version and Burke's Peerage led to a scandal that caused him to flee to India. There he involved himself in monastic life and wrote on aspects of Buddhism. He died in the Punjab in 1962 aged 47. 